\title{
Integral relations and the adiabatic expansion method for $1+2$ reactions above the breakup threshold: Helium trimers with soft-core potentials
}

\author{
E. Garrido \\ Instituto de Estructura de la Materia, CSIC, Serrano 123, E-28006 Madrid, Spain \\ C. Romero-Redondo \\ TRIUMF, 4004 Wesbrook Mall, Vancouver, British Columbia, Canada V6T 2A3 \\ A. Kievsky and M. Viviani \\ Istituto Nazionale di Fisica Nucleare, Largo Pontecorvo 3, 56100 Pisa, Italy
}

(Received 19 October 2012; published 26 November 2012)

\begin{abstract}
The integral relations formalism introduced in [P. Barletta et al., Phys. Rev. Lett. 103, 090402 (2009); C. Romero-Redondo et al., Phys. Rev. A 83, 022705 (2011)] and designed to describe $1+N$ reactions is extended here to collision energies above the threshold for the target breakup. These two relations are completely general, and in this work they are used together with the adiabatic expansion method for the description of $1+2$ reactions. The neutron-deuteron breakup, for which benchmark calculations are available, is taken as a test of the method. The $s$-wave collision between the ${ }^{4} \mathrm{He}$ atom and ${ }^{4} \mathrm{He}_{2}$ dimer above the breakup threshold and the possibility of using soft-core two-body potentials plus a short-range three-body force will be investigated. Comparisons to previous calculations for the three-body recombination and collision dissociation rates will be shown.
\end{abstract}

DOI: 10.1103/PhysRevA.86.052709

PACS number(s): 03.65.Nk, 21.45.-v, 31.15.xj, 34.50.-s

\section{INTRODUCTION}

Calculation of continuum states corresponding to processes where a particle hits a bound $N$-body system requires, in principle, knowledge of the corresponding $(1+N)$-body wave function at large distances. Needless to say, the technical difficulties one has to face in order to obtain the wave function increase dramatically with $N$. In fact, already for $N=2$, calculation of the three-body wave function is far from being trivial.

However, even if knowledge of the $(1+N)$-body wave function is unavoidable, the possibility of reducing the distance at which such a wave function is needed is in itself an important step forward in the description of the reaction. As shown in $[1,2]$, this can indeed be done by means of two integral relations that are based on the Kohn variational principle. These two integral relations are a generalization to more than two particles of the integral relation given in [3,4], and they permit us to obtain the $\mathcal{K}$ (or $\mathcal{S}$ ) matrix of the reaction by using only the internal part of the wave function. Therefore, all the physical information concerning a given $1+N$ reaction can be obtained without an accurate knowledge of the asymptotic part of the wave function.

The fact that the asymptotic part is not needed any longer leads to a drastic reduction of the computer effort required to extract the $\mathcal{K}$ matrix. The only condition necessary to obtain accurate second-order estimates of the $\mathcal{K}$ matrix through the integral relations is that the trial wave function must fulfill the Schrödinger equation only in the interaction region. This means that, for instance, scattering states can be described with bound-state-like trial wave functions [5].

In $[1,2]$ the integral relations were implemented to describe $1+2$ reactions below the breakup threshold. When used in combination with the hyperspherical adiabatic expansion method the dimension of the $\mathcal{K}$ matrix describing the reaction is dictated by the number of adiabatic potentials related to the possible outgoing elastic or inelastic channels, which typically is very small. Furthermore, thanks to integral relations, the convergence of the $\mathcal{K}$ matrix in terms of the adiabatic channels included in the expansion of the wave function is highly accelerated. Actually, the pattern of convergence is similar to the one found when the same method is used for the description of bound states [1,2]. This is, in fact, not a minor issue since, as proved in [6], when used to describe low-energy scattering states, the convergence of the adiabatic expansion slows down significantly, even to the point that an accurate calculation of the $\mathcal{K}$ matrix would require infinitely many adiabatic terms in the expansion, which in practice makes the procedure useless.

The success of the method for energies below the breakup threshold immediately suggests its extension to describe lowenergy breakup reactions. In this case the dimension of the $\mathcal{K}$ matrix is not finite since, contrary to the elastic and inelastic channels or transfer reactions, the full three-body continuum states are described by infinitely many adiabatic potentials.

The first goal of this work is to generalize the method described in $[1,2]$ for $1+N$ reactions to energies above the threshold for breakup of the bound target. This generalization will be shown in Sec. II, where a short summary of the method described in [2] will be given. In Sec. III, the $n$ - $d$ reaction, for which a series of benchmark calculations are available $[7,8]$, will be used as a test of the method.

The second goal, which will be discussed in Sec. IV, concerns the use of the new method to investigate the ${ }^{4} \mathrm{He}+{ }^{4} \mathrm{He}_{2}$ atomic reaction above the dimer breakup threshold. The case of the $0^{+}$state will be considered. In particular, we will focus on the use of soft-core ${ }^{4} \mathrm{He}-{ }^{4} \mathrm{He}$ potentials. As shown in [9], the use of an attractive Gaussian potential reproducing the same two-body properties as a standard hard-core potential (for instance, the second mimic of the second Liu and McLean (LM2M2) potential) leads to equivalent bound three-body 
systems and phase shifts for the elastic ${ }^{4} \mathrm{He}+{ }^{4} \mathrm{He}_{2}$ reaction, but only once that the soft-core potential is used together with a three-body short-term force. The possibility of using the same kind of potential also to describe the breakup channel is interesting since it automatically eliminates all the technical difficulties arising from the presence of a hard-core repulsion in the potential (see, for instance, Ref. [10]). Finally, we close this work with a short summary and the conclusions.

\section{FORMALISM}

In Refs. [1,2] $1+2$ reactions were described within the framework of the hyperspherical adiabatic expansion method for energies below the threshold for breakup of the dimer. Therefore, only elastic, inelastic, and transfer processes were possible. Since the formalism is described in great detail in Ref. [2], here we just summarize its main aspects, which are given in the first part of this section. In particular, we will focus on those key points that permit an easier understanding of the generalization of the method to energies above the two-body breakup threshold, which will be shown in the second part of this section. In the last part we will describe the integral relations, which are actually the tools that permit us to extract the $\mathcal{K}$ matrix of the reaction from the internal part of the wave functions.

\section{A. Sketch of the method for energies below the breakup threshold}

Given a three-body system, the corresponding wave function within the frame of the adiabatic expansion method is written as

$$
\Psi(\boldsymbol{x}, \boldsymbol{y})=\frac{1}{\rho^{5}} \sum_{n=1}^{\infty} f_{n}(\rho) \Phi_{n}(\rho, \Omega),
$$

where $\boldsymbol{x}$ and $\boldsymbol{y}$ are the usual Jacobi coordinates and $\{\rho, \Omega\}$ are the hyperradius and the five hyperangles obtained from $\boldsymbol{x}$ and $\boldsymbol{y}$ [11]. The wave function has a well-defined total angular momentum $J$, but for simplicity in the notation we omit this index (and its projection) in the expression above.

In hyperspherical coordinates the Hamiltonian operator $\hat{\mathcal{H}}$ takes the form

$$
\hat{\mathcal{H}}=-\frac{\hbar^{2}}{2 m} \hat{T}_{\rho}+\hat{\mathcal{H}}_{\Omega}
$$

where $\hat{T}_{\rho}$ is the hyperradial kinetic energy operator, $\hat{\mathcal{H}}_{\Omega}$ contains all the dependence on the hyperangles, and $m$ is an arbitrary normalization mass. The angular functions $\Phi_{n}(\rho, \Omega)$, which form the complete basis used for the wave-function expansion (1), are actually the eigenfunctions of $\hat{\mathcal{H}}_{\Omega}$,

$$
\hat{\mathcal{H}}_{\Omega} \Phi_{n}(\rho, \Omega)=\frac{\hbar^{2}}{2 m} \frac{1}{\rho^{2}} \lambda_{n}(\rho) \Phi_{n}(\rho, \Omega),
$$

and the adiabatic effective potentials

$$
V_{e f f}^{(n)}(\rho)=\frac{\hbar^{2}}{2 m}\left(\frac{\lambda_{n}(\rho)+\frac{15}{4}}{\rho^{2}}-Q_{n n}(\rho)\right)
$$

enter in the coupled set of radial equations that permit us to obtain the radial functions $f_{n}(\rho)$,

$$
\begin{aligned}
& \left\{-\frac{d^{2}}{d \rho^{2}}+\frac{2 m}{\hbar^{2}}\left[V_{e f f}^{(n)}(\rho)-E\right]\right\} f_{n}(\rho) \\
& +\sum_{n^{\prime} \neq n}\left(-2 P_{n n^{\prime}} \frac{d}{d \rho}-Q_{n n^{\prime}}\right) f_{n^{\prime}}(\rho)=0,
\end{aligned}
$$

where

$$
\begin{gathered}
Q_{n n^{\prime}}(\rho)=\left\langle\Phi_{n}(\rho, \Omega)\left|\frac{\partial^{2}}{\partial \rho^{2}}\right| \Phi_{n^{\prime}}(\rho, \Omega)\right\rangle_{\Omega}, \\
P_{n n^{\prime}}(\rho)=\left\langle\Phi_{n}(\rho, \Omega)\left|\frac{\partial}{\partial \rho}\right| \Phi_{n^{\prime}}(\rho, \Omega)\right\rangle_{\Omega},
\end{gathered}
$$

where the subscript $\Omega$ indicates integration over the hyperangles only (see [11] for details).

A typical behavior of the adiabatic potentials is shown in Fig. 1. They correspond to a three-body system where two of the two-body subsystems have a bound state. This is reflected in the fact that the two lowest effective adiabatic potentials go asymptotically to the binding energies $E_{2 b}^{(1)}$ and $E_{2 b}^{(2)}$ of each bound two-body system.

In Fig. 1 the different regions defined by the energy of the incident particles are depicted. All the three-body energies $E$ such that $E_{2 b}^{(1)}<E<E_{2 b}^{(2)}$ (such as $E^{(1)}$ in Fig. 1) correspond to processes where only one channel is open. Only the elastic collision between the third particle and the bound two-body state with energy $E_{2 b}^{(1)}$ is possible. When the three-body energy increases up to the region $E_{2 b}^{(2)}<E<0$ ( $E^{(2)}$ in Fig. 1), a second channel is open. Two different collisions are now possible, the one where a particle hits the bound state with binding energy $E_{2 b}^{(1)}$ and the one where a particle hits the state with binding energy $E_{2 b}^{(2)}$. In the same way, each of these reactions has two possible outgoing channels, corresponding to the two allowed bound two-body states and the third particle in the continuum. In other words, in this energy range the

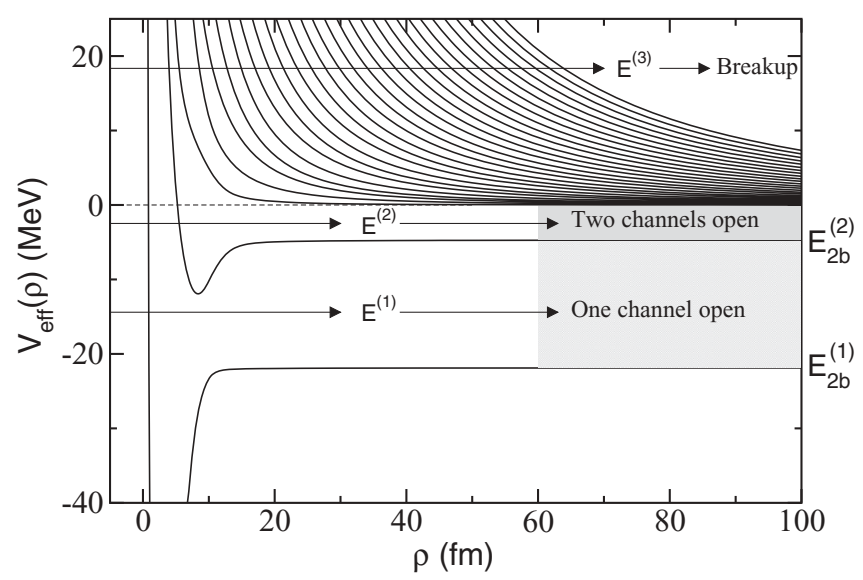

FIG. 1. Typical effective adiabatic potentials for a three-body system where two two-body bound states are present. The two lowest adiabatic potentials go asymptotically to the binding energies $E_{2 b}^{(1)}$ and $E_{2 b}^{(2)}$ of the two-body bound states. For a given three-body energy $E$, when $E_{2 b}^{(1)}<E<E_{2 b}^{(2)}$, only one channel is open; when $E_{2 b}^{(2)}<E<0$, both channels are open, and for $E>0$ the breakup channel is also open. 
inelastic (if the two bound two-body states correspond to the same subsystem) or transfer (if the two bound two-body states correspond to different subsystems) channel is open. When $E>0$ (such as $E^{(3)}$ in Fig. 1), the breakup channel is also open, and it is described by the remaining infinitely many adiabatic potentials.

The coupled system of radial equations (5) decouples asymptotically, and each of the radial wave functions behave at large distances as dictated by

$$
\left(-\frac{\hbar^{2}}{2 m} \frac{d^{2}}{d \rho^{2}}+V_{e f f}^{(n)}(\rho)-E\right) f_{n}(\rho)=0 .
$$

When $n$ corresponds to a closed channel, the radial wave function $f_{n}$ vanishes asymptotically. For values of $E$ below the two-body breakup threshold, as considered in [2], and for a given incoming $1+2$ channel, only outgoing $1+2$ channels are allowed (either elastic, inelastic, or transfer). In this case, as shown in [11], the equation above describing the asymptotic behavior of the open-channel wave functions becomes

$$
\left[\frac{d^{2}}{d y_{n}^{2}}+\left(k_{y}^{(n)}\right)^{2}-\frac{\ell_{y}\left(\ell_{y}+1\right)}{y_{n}^{2}}\right] f_{n}(\rho)=0,
$$

where $\ell_{y}$ is the relative angular momentum between the dimer and the third particle, $y_{n}$ refers to the modulus of the Jacobi coordinate between the center of mass of the outgoing bound two-body system and the third particle, and

$$
k_{y}^{(n)}=\sqrt{\frac{2 m}{\hbar^{2}}\left(E-E_{2 b}^{(n)}\right)},
$$

with $E_{2 b}^{(n)}$ being the binding energy of the bound two-body system associated with the open channel $n$.

With this in hand, it is not difficult to see [2] that the asymptotic form of the corresponding three-body wave function is given by

$$
\Psi_{i} \rightarrow \sum_{n=1}^{n_{0}}\left(A_{i n}^{(K)} F_{n}^{(K)}+B_{i n}^{(K)} G_{n}^{(K)}\right),
$$

where $i$ refers to the incoming channel, $n_{0}$ is the number of open channels (all of them $1+2$ channels), and

$$
\begin{aligned}
F_{n}^{(K)} & =\sqrt{k_{y}^{(n)}} j_{\ell_{y}}\left(k_{y}^{(n)} y_{n}\right) \frac{1}{\rho^{3 / 2}} \Phi_{n}(\rho, \Omega), \\
G_{n}^{(K)} & =\sqrt{k_{y}^{(n)}} \eta_{\ell_{y}}\left(k_{y}^{(n)} y_{n}\right) \frac{1}{\rho^{3 / 2}} \Phi_{n}(\rho, \Omega),
\end{aligned}
$$

where $j_{\ell}$ and $\eta_{\ell}$ are the usual regular and irregular Bessel functions (provided that we are dealing with short-range potentials).

As shown in [2], Eq. (11) can be written in a compact matrix form as

$$
\Psi \rightarrow A^{(K)} F^{(K)}+B^{(K)} G^{(K)}=A^{(K)}\left(F^{(K)}-\mathcal{K} G^{(K)}\right),
$$

where $\Psi$ is a column vector with $n_{0}$ terms corresponding to the $n_{0}$ open channels, $A^{(K)}$ and $B^{(K)}$ are $n_{0} \times n_{0}$ matrices made by the $A_{i n}^{(K)}$ and $B_{i n}^{(K)}$ elements in Eq. $(11)$, and $F^{(K)}$ and $G^{(K)}$ are again column vectors whose $n_{0}$ terms are given by Eqs. (12) and (13). From the equation above it is clear that the $\mathcal{K}$ matrix of the reaction is given by

$$
\mathcal{K}=-A^{(K)^{-1}} B^{(K)} \text {. }
$$

This matrix is real, and from it one can easily obtain the $\mathcal{S}$ matrix as $(1+i \mathcal{K})(1-i \mathcal{K})^{-1}$.

\section{B. Generalization to energies above the breakup threshold}

When the total three-body energy $E$ in a $1+2$ reaction is above the threshold for breakup of the dimer, the first consequence is that infinitely many adiabatic channels are then open (see Fig. 1 for $E=E^{(3)}$ ). Of course, still a finite number of them correspond to elastic, inelastic, or transfer processes, and the infinitely many remaining ones describe the breakup channel. Therefore, in this case the corresponding $\mathcal{K}$ (or $\mathcal{S}$ ) matrix has an infinite dimension.

In any case, for the finite open channels corresponding to outgoing $1+2$ structures, the expressions given in the previous subsection are still valid. This means that for these particular outgoing channels the three-body wave function behaves asymptotically as given by Eq. (11), and Eqs. (12) and (13) are still valid.

On the other hand, the breakup channels are characterized by the fact that the effective potentials $V_{e f f}^{(n)}$ associated with them go asymptotically to zero as

$$
V_{\text {eff }}^{(n)}(\rho) \stackrel{\rho \rightarrow \infty}{\rightarrow} \frac{\hbar^{2}}{2 m} \frac{\left(K+\frac{3}{2}\right)\left(K+\frac{5}{2}\right)}{\rho^{2}},
$$

where $K$ is the grand-angular quantum number defined as $2 v+\ell_{x}+\ell_{y}$, where $\ell_{x}$ and $\ell_{y}$ are the orbital angular momenta associated with the Jacobi coordinates $x$ and $y$, respectively, and $v=0,1,2, \ldots$ Therefore, asymptotically, each breakup adiabatic potential is associated with a fixed value of $K$. In fact, the corresponding angular eigenfunction $\Phi_{n}(\rho, \Omega)$ is, also asymptotically, a linear combination of hyperspherical harmonics with that particular value of $K$.

When inserting (16) into (8), we easily obtain that, asymptotically, the radial wave function for an outgoing breakup channel $n$ satisfies the equation

$$
\left[\frac{d^{2}}{d \rho^{2}}+\kappa^{2}-\frac{\left(K+\frac{3}{2}\right)\left(K+\frac{5}{2}\right)}{\rho^{2}}\right] f_{n}(\rho)=0,
$$

which is formally identical to the Eq. (9), which is satisfied by the radial wave functions associated with outgoing $1+2$ channels, except replacing $\ell_{y}$ by $K+3 / 2, k_{y}^{(n)}$ by $\kappa=$ $\sqrt{2 m E / \hbar^{2}}$, and $y_{n}$ by $\rho$.

Therefore, in the more general case where the breakup channel is open and assuming an incoming $1+2$ channel $i$, the asymptotic form of the corresponding three-body wave function is given by

$$
\Psi_{i} \rightarrow \sum_{n=1}^{\infty}\left(A_{i n}^{(K)} F_{n}^{(K)}+B_{i n}^{(K)} G_{n}^{(K)}\right),
$$

where $F_{n}^{(K)}$ and $G_{n}^{(K)}$ are still given by Eqs. (12) and (13) but, of course, with the understanding that when $n$ corresponds to an outgoing breakup channel, the replacements $\ell_{y} \rightarrow K+$ $3 / 2, k_{y}^{(n)} \rightarrow \kappa$, and $y_{n} \rightarrow \rho$ have to be made [note that the relations $j_{K+\frac{3}{2}}(z)=\sqrt{\frac{\pi}{2 z}} J_{K+2}(z)$ and $\eta_{K+\frac{3}{2}}(z)=\sqrt{\frac{\pi}{2 z}} Y_{K+2}(z)$ permit us to write Eqs. (12) and (13) in terms of the Bessel functions $J_{K+2}$ and $Y_{K+2}$, which is how the asymptotic form of the breakup channels is usually presented in the literature]. 
Of course, the matrix form in Eq. (14) of the asymptotic wave function can still be used. As before, the $\mathcal{K}$ matrix of the reaction is given by $\mathcal{K}=-A^{(K)^{-1}} B^{(K)}$, but now matrices $A^{(K)}$ and $B^{(K)}$ have, in principle, an infinite dimension, and some truncation is then required.

Due to the fact that the hyperradius $\rho$ appears as the natural radial coordinate describing the asymptotic behavior of the breakup channels, we then have that, for these channels, the adiabatic expansion is able to reach the correct asymptotic behavior for a sufficiently large, but finite, value of $\rho$. For this reason, for processes without $1+2$ open channels $(3 \rightarrow 3$ processes), the $\mathcal{K}$ matrix could, in principle, be extracted with sufficient accuracy from the asymptotic behavior. However, this is not true when $1+2$ channels are open. For these channels the correct asymptotic form [described by (12) and (13)] cannot be reached until the Jacobi coordinate $y$ and hyperradius $\rho$ are equal, which only happens at infinity. Therefore it is essential in this case to use a formalism in which the $\mathcal{K}$ matrix is not extracted from the asymptotic part of the wave function but from its internal part.

\section{Integral relations}

The derivation of the integral relations has been shown in Ref. [2]. This derivation is completely general, and it is not particularized to the case of incident energies below the breakup threshold. Therefore, the same expressions derived in [2] apply when the breakup channel is open. According to it, by making use of the Kohn variational principle, it has been proved that when using a trial three-body wave function $\Psi^{t}$, one can obtain the matrices $A^{(K)}$ and $B^{(K)}$ accurate up to second order in $\delta\left(\Psi-\Psi^{t}\right)$, and their matrix elements are given by

$$
\begin{aligned}
B_{i j} & =\frac{2 m}{\hbar^{2}}\left\langle\Psi_{i}^{t}|\hat{\mathcal{H}}-E| F_{j}^{(K)}\right\rangle, \\
A_{i j} & =-\frac{2 m}{\hbar^{2}}\left\langle\Psi_{i}^{t}|\hat{\mathcal{H}}-E| G_{j}^{(K)}\right\rangle,
\end{aligned}
$$

where $\Psi_{i}^{t}$ describes each possible incoming channel and the index $j$ refers to each possible outgoing channel (either $1+2$ or breakup).

In this work, the trial three-body wave function will be the one obtained as sketched in Sec. II A. To be precise, it will be obtained by solving the Faddeev equations by means of the hyperspherical adiabatic expansion method (see Ref. [11] for details).

Since the regular and irregular functions $F^{(K)}$ and $G^{(K)}$ are asymptotically solutions of $(\mathcal{H}-E) F^{(K)}, G^{(K)}=0$, it is then clear that integral relations (19) and (20) depend only on the short-range structure of the scattering wave function $\Psi^{t}$. It is important to note that the function $G^{(K)}$, defined in Eq. (13), is irregular at the origin. In order to avoid the problems arising from this fact, the $G^{(K)}$ function is regularized. This means that in Eq. (20) the Bessel function $\eta$ contained in $G^{(K)}$ is actually replaced by another function that goes to zero at the origin and behaves exactly as $\eta$ at large distances. In [2] this was done by using $\tilde{\eta}_{\ell}(z)=\left(1-e^{-\gamma z}\right)^{\ell+1} \eta_{\ell}(z)$, where $\gamma(>0)$ is a parameter. However, in our case, where index $\ell$ can reach pretty high values ( $\ell=K+3 / 2$ in the breakup channels), this procedure is not appropriate.
In this work we have regularized the irregular Bessel function by solving Eq. (8) for each individual adiabatic potential. The solutions of this equation behave asymptotically as $f_{n}(z) \rightarrow z j_{v}(z)-\tan \delta_{n} z \eta_{\nu}(z)$, where $\delta_{n}$ is the phase shift, and index $v$ is equal to $\ell_{y}$ for the $1+2$ channels and $K+3 / 2$ for the breakup channels. Then, the irregular Bessel function implicitly contained in Eq. (20) is replaced by

$$
\tilde{\eta}_{\nu}(z)=\frac{z j_{v}(z)-f_{n}(z)}{z \tan \delta_{n}},
$$

which by construction is regular at the origin and goes asymptotically to $\eta_{v}(z)$.

\section{A TEST CASE: NEUTRON-DEUTERON SCATTERING}

The benchmark solutions for neutron-deuteron breakup amplitudes are shown in $[7,8]$. For this reason we take this case as a test for the method shown in this work. In particular, the nucleon-nucleon interaction is chosen to be the revised Malfliet-Tjon I-III model $s$-wave potential [7], which for the spin triplet and singlet cases takes the form

$$
\begin{aligned}
& V_{t}(r)=\frac{1}{r}\left(-626.885 e^{-1.55 r}+1438.72 e^{-3.11 r}\right), \\
& V_{s}(r)=\frac{1}{r}\left(-513.968 e^{-1.55 r}+1438.72 e^{-3.11 r}\right),
\end{aligned}
$$

where $r$ is given in fermi (fm), and the potential is given in MeV. Also, $\hbar^{2} / m=41.47 \mathrm{MeV} \mathrm{fm}{ }^{2}$. The potential above leads to a binding energy for the deuteron of $2.2307 \mathrm{MeV}$.

In the calculation only $s$ waves are considered. Therefore, two different total angular momenta are possible, the quartet case $(J=3 / 2)$, for which only the triplet $s$-wave potential (22) enters, and the doublet case $(J=1 / 2)$, for which both the singlet and the triplet potentials contribute. In Fig. 2 we show the computed adiabatic effective potentials [Eq. (8)] for the doublet case. As you can see, they follow the general trend of the potentials shown in Fig. 1, although in this case there is only one $1+2$ channel, which corresponds to the neutron-deuteron reaction that we want to investigate.

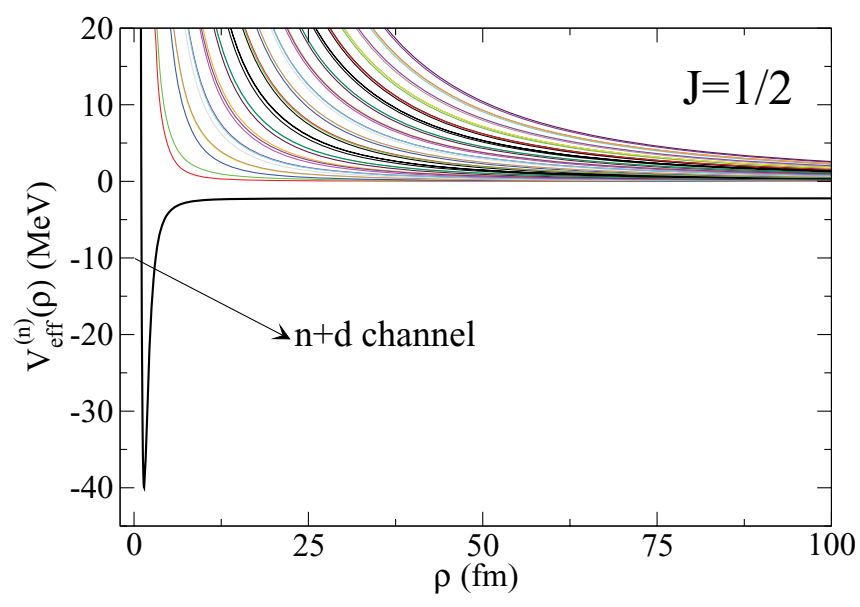

FIG. 2. (Color online) Effective adiabatic potentials for the neutron-neutron-proton system in the doublet case. The thick curve corresponds to the neutron + deuteron channel, and it goes asymptotically to the deuteron binding energy. All the remaining adiabatic potentials correspond to breakup channels. 
The corresponding adiabatic potential is given by the thick solid line in Fig. 2, and it will be labeled as channel 1. As expected, the asymptotic value of this potential corresponds to the binding energy of the deuteron.

\section{A. Inelasticity and phase shifts}

The unitarity of the $\mathcal{S}$ matrix implies that given an incoming channel, for instance, channel $1(n+d$ channel), we have that $\sum_{1}^{\infty}\left|\mathcal{S}_{1 n}\right|^{2}=1$, or, in other words,

$$
\sum_{n=2}^{\infty}\left|\mathcal{S}_{1 n}\right|^{2}=1-\left|\mathcal{S}_{11}\right|^{2},
$$

which means that an accurate calculation of the elastic term $\mathcal{S}_{11}$ amounts to an accurate calculation of the infinite summation of the $\left|\mathcal{S}_{1 n}\right|^{2}$ terms $(n>1)$ corresponding the breakup channels. Also, the complex value of $\mathcal{S}_{11}$ can be written in terms of a complex phase shift $\delta$ as

$$
\mathcal{S}_{11}=e^{2 i \delta}=e^{-2 \operatorname{Im}(\delta)} e^{2 i \operatorname{Re}(\delta)}=\left|\mathcal{S}_{11}\right| e^{2 i \operatorname{Re}(\delta)} .
$$

The value of $\left|\mathcal{S}_{11}\right|^{2}$ gives the probability of elastic neutrondeuteron scattering, and $\left|\mathcal{S}_{11}\right|$ is usually referred to as the inelasticity parameter (denoted by $\eta$ in $[7,8]$ ). Obviously, the closer the inelasticity is to 1 , the more elastic the reaction is. In fact, for energies below the breakup threshold the phase shift is real and $\left|\mathcal{S}_{11}\right|=1$.

In Table I we give the inelasticity parameter $\left|\mathcal{S}_{11}\right|$ for the two laboratory neutron energies used in [8], i.e., 14.1 and 42.0 MeV. We have computed the inelasticity parameter for both the doublet and the quartet states. We give the computed values of $\left|\mathcal{S}_{11}\right|$ for different truncations in the infinite summation (18). In particular, in Table I we give the value $K_{\max }$

TABLE I. Inelasticity parameter $\left|\mathcal{S}_{11}\right|$ for the neutron-deuteron scattering for two different laboratory neutron beam energies (14.1 and $42.0 \mathrm{MeV}$ ) for the doublet and quartet cases. The value of $K_{\max }$ is the $K$ value associated with the last adiabatic potential included in the calculation. The numbers within parentheses have been obtained without use of the integral relations. The last row gives the value quoted in Ref. [8].

\begin{tabular}{lccccc}
\hline \hline & \multicolumn{2}{c}{ Doublet } & & \multicolumn{2}{c}{ Quartet } \\
\cline { 2 - 3 } \cline { 5 - 6 }$K_{\max }$ & $14.1 \mathrm{MeV}$ & $42.0 \mathrm{MeV}$ & & $14.1 \mathrm{MeV}$ & $42.0 \mathrm{MeV}$ \\
\hline \multirow{2}{*}{4} & $\mathbf{0 . 4 6 6 2}$ & $\mathbf{0 . 4 9 2 9}$ & $\mathbf{0 . 9 7 9 4}$ & $\mathbf{0 . 8 9 7 5}$ \\
& $(0.4710)$ & $(0.4719)$ & & $(0.9809)$ & $(0.8865)$ \\
8 & $\mathbf{0 . 4 6 3 7}$ & $\mathbf{0 . 4 9 9 3}$ & $\mathbf{0 . 9 7 8 4}$ & $\mathbf{0 . 9 0 2 6}$ \\
& $(0.4670)$ & $(0.4985)$ & & $(0.9794)$ & $(0.9050)$ \\
12 & $\mathbf{0 . 4 6 4 0}$ & $\mathbf{0 . 5 0 1 4}$ & $\mathbf{0 . 9 7 8 3}$ & $\mathbf{0 . 9 0 3 0}$ \\
& $(0.4664)$ & $(0.5041)$ & $(0.9792)$ & $(0.9071)$ \\
16 & $\mathbf{0 . 4 6 4 3}$ & $\mathbf{0 . 5 0 1 9}$ & $\mathbf{0 . 9 7 8 2}$ & $\mathbf{0 . 9 0 3 1}$ \\
& $(0.4666)$ & $(0.5051)$ & $(0.9792)$ & $(0.9071)$ \\
20 & $\mathbf{0 . 4 6 4 4}$ & $\mathbf{0 . 5 0 2 1}$ & $\mathbf{0 . 9 7 8 2}$ & $\mathbf{0 . 9 0 3 3}$ \\
& $(0.4666)$ & $(0.5052)$ & & $(0.9791)$ & $(0.9069)$ \\
24 & $\mathbf{0 . 4 6 4 5}$ & $\mathbf{0 . 5 0 2 2}$ & $\mathbf{0 . 9 7 8 2}$ & $\mathbf{0 . 9 0 3 3}$ \\
& $(0.4666)$ & $(0.5055)$ & $(0.9790)$ & $(0.9071)$ \\
28 & $\mathbf{0 . 4 6 4 5}$ & $\mathbf{0 . 5 0 2 2}$ & $\mathbf{0 . 9 7 8 2}$ & $\mathbf{0 . 9 0 3 3}$ \\
& $(0.4667)$ & $(0.5056)$ & $(0.9790)$ & $(0.9071)$ \\
Ref. $[8]$ & $\mathbf{0 . 4 6 4 9}$ & $\mathbf{0 . 5 0 2 2}$ & $\mathbf{0 . 9 7 8 2}$ & $\mathbf{0 . 9 0 3 3}$ \\
\hline \hline
\end{tabular}

of the asymptotic grand-angular quantum number associated with the last adiabatic potential included in the calculation [see Eq. (16)]. The values given in Table I without parentheses have been obtained by using integral relations (19) and (20), while the ones within parentheses have been calculated from the $A^{(K)}$ and $B^{(K)}$ matrices extracted directly from the asymptotic part of the three-body wave function, as indicated in Eq. (14). The last row in Table I gives the value obtained in [8].

As seen in Table I, when using the integral relations, the agreement with the results in [8] is very good. Actually, we obtain precisely the same result for the two energies in the quartet case, and a tiny difference clearly smaller than $0.1 \%$ in the doublet case. Furthermore, the pattern of convergence is rather fast, especially in the quartet case, for which already for $K_{\max }=8$ we obtain a result that can be considered very accurate. In the doublet case the convergence is a bit slower, and a value of $K_{\max }$ of about 16 is needed. As we can see from the values within parenthesis, when the integral relations are not used, the value of $\left|\mathcal{S}_{11}\right|$ seems to converge more slowly, and even if converged, the result is less accurate.

From Eq. (25) we have that, together with the inelasticity parameter $\left|\mathcal{S}_{11}\right|$, a complete specification of the matrix element $\mathcal{S}_{11}$ requires knowledge of the real part of the phase shift $\operatorname{Re}(\delta)$. The corresponding computed values are shown in Table II, where the meaning of the different columns is the same as in Table I. The behavior of $\operatorname{Re}(\delta)$ is similar to that shown in Table I for $\left|\mathcal{S}_{11}\right|$. For the quartet case the same results as in [8] are obtained, while for the doublet a very small difference smaller than $0.1 \%$ is again found. The pattern of convergence is also similar. A $K_{\max }$ value of 12 is already enough to get a quite accurate value. The main difference compared to the results for $\left|\mathcal{S}_{11}\right|$ shown in Table I is that now the values of $\operatorname{Re}(\delta)$ obtained without using the integral relations are much less converged and much less accurate. The difference with the true result can reach up to $10 \%$. This behavior was already observed in [1] for the phase shift in a reaction below the breakup threshold. This is due to the fact that the hyperradius $\rho$ and the Jacobi coordinate $y$ entering in the asymptotic forms

TABLE II. The same as Table I for $\operatorname{Re}(\delta)$.

\begin{tabular}{lccccc}
\hline \hline & \multicolumn{2}{c}{ Doublet } & & \multicolumn{2}{c}{ Quartet } \\
\cline { 2 - 3 } \cline { 5 - 6 }$K_{\max }$ & $14.1 \mathrm{MeV}$ & $42.0 \mathrm{MeV}$ & & $14.1 \mathrm{MeV}$ & $42.0 \mathrm{MeV}$ \\
\hline 4 & $\mathbf{1 0 5 . 8 2}$ & $\mathbf{4 2 . 6 6}$ & $\mathbf{6 9 . 0 4}$ & $\mathbf{3 8 . 9 8}$ \\
& $(97.62)$ & $(28.89)$ & & $(60.88)$ & $(25.29)$ \\
8 & $\mathbf{1 0 5 . 5 7}$ & $\mathbf{4 1 . 6 5}$ & $\mathbf{6 8 . 9 9}$ & $\mathbf{3 7 . 9 5}$ \\
& $(99.91)$ & $(32.88)$ & & $(63.24)$ & $(28.98)$ \\
12 & $\mathbf{1 0 5 . 5 3}$ & $\mathbf{4 1 . 4 9}$ & & $\mathbf{6 8 . 9 8}$ & $\mathbf{3 7 . 7 7}$ \\
& $(101.01)$ & $(34.80)$ & & $(64.43)$ & $(31.07)$ \\
16 & $\mathbf{1 0 5 . 5 3}$ & $\mathbf{4 1 . 4 6}$ & & $\mathbf{6 8 . 9 7}$ & $\mathbf{3 7 . 7 3}$ \\
& $(101.68)$ & $(36.05)$ & & $(65.11)$ & $(32.28)$ \\
20 & $\mathbf{1 0 5 . 5 3}$ & $\mathbf{4 1 . 4 5}$ & & $\mathbf{6 8 . 9 6}$ & $\mathbf{3 7 . 7 2}$ \\
& $(102.12)$ & $(36.81)$ & & $(65.54)$ & $(33.03)$ \\
24 & $\mathbf{1 0 5 . 5 3}$ & $\mathbf{4 1 . 4 4}$ & $\mathbf{6 8 . 9 6}$ & $\mathbf{3 7 . 7 1}$ \\
& $(102.41)$ & $(37.30)$ & & $(65.84)$ & $(33.55)$ \\
28 & $\mathbf{1 0 5 . 5 3}$ & $\mathbf{4 1 . 4 4}$ & $\mathbf{6 8 . 9 6}$ & $\mathbf{3 7 . 7 1}$ \\
& $(102.49)$ & $(37.90)$ & & $(65.88)$ & $(33.58)$ \\
Ref. $[8]$ & $\mathbf{1 0 5 . 5 0}$ & $\mathbf{4 1 . 3 7}$ & $\mathbf{6 8 . 9 6}$ & $\mathbf{3 7 . 7 1}$ \\
\hline \hline
\end{tabular}


TABLE III. Helium dimer and helium trimer properties obtained with the soft-core potential in Eq. (26), the hard-core potential LM2M2 in Ref. [14], the modified soft-core potential (soft core 2) in Eq. (30), and the hard-core SAPT potential in Ref. [17]. The two-body properties are given in the top part of the table, where $E_{2 b}, a, r_{0}$, and $\langle r\rangle$ are the dimer binding energy, the scattering length, the effective range, and the average interatomic distance, respectively. The three-body properties are given in the bottom part, where $E_{3 b}^{(\text {g.s. })}, E_{3 b}^{(e x c .)}$, and $a_{0}$ are the binding energy of the trimer ground state, the binding energy of the trimer excited state, and the atom-dimer scattering length, respectively. For the soft-core and soft-core 2 potentials the column corresponding to the trimer properties has been split in two. The results on the left have been obtained without including any effective three-body force. The ones on the right are obtained after including a three-body force fitted to match the binding energy of the trimer ground state obtained with the LM2M2 [19] and SAPT [17,20] potentials, respectively. The parameters used for the three-body potential are denoted by $W_{0}$ and $\rho_{0}$ [see Eq. (27)]. All the distances are given in a.u. and the energies are given in mK, except $W_{0}$, which is given in $\mathrm{K}$.

\begin{tabular}{|c|c|c|c|c|c|c|}
\hline & \multicolumn{2}{|c|}{ Soft core } & LM2M2 [19] & \multicolumn{2}{|c|}{ Soft core 2} & SAPT $[17,20]$ \\
\hline$E_{2 b}(\mathrm{mK})$ & \multicolumn{2}{|c|}{-1.296} & -1.302 & \multicolumn{2}{|c|}{-1.554} & -1.564 \\
\hline$a$ (a.u.) & \multicolumn{2}{|c|}{189.95} & 189.05 & \multicolumn{2}{|c|}{174.09} & 173.50 \\
\hline$r_{0}$ (a.u.) & \multicolumn{2}{|c|}{13.85} & 13.84 & \multicolumn{2}{|c|}{13.80} & 13.79 \\
\hline \multirow[t]{3}{*}{$\langle r\rangle$ (a.u.) } & & & 98.2 & & & 90.3 \\
\hline & \multicolumn{2}{|c|}{ Soft core } & \multicolumn{4}{|c|}{ Soft core 2} \\
\hline & No 3b-force & $3 b$-force & LM2M2 [19] & No 3b-force & $3 b$-force & SAPT $[17,20]$ \\
\hline$\overline{\left(W_{0}, \rho_{0}\right)(\mathrm{K}, \text { a.u. })}$ & $(0,-)$ & $(18.314,6)$ & & $(0,-)$ & $(17.760,6)$ & \\
\hline$E_{3 b}^{(g . s .)}(\mathrm{mK})$ & -150.0 & -126.4 & -126.4 & -154.9 & -130.9 & -130.9 \\
\hline$E_{3 b}^{(e x c .)}(\mathrm{mK})$ & -2.467 & -2.287 & -2.265 & -2.805 & -2.612 & -2.588 \\
\hline$a_{0}$ (a.u.) & 165.9 & 210.6 & 224.3 & 181.7 & 226.0 & 226.8 \\
\hline
\end{tabular}

(12) and (13) are equivalent only at infinity, as noted at the end of Sec. II B. This means that a correct extraction of the phase shift from the asymptotic part of the wave function requires us to impose the boundary condition at infinity, for which infinite adiabatic terms would also be needed.

Therefore, for energies above the breakup threshold, the conclusion from Tables I and II is similar to the one reached in [1] for $1+2$ elastic processes, that is, the use of the integral relations is crucial from two different points of view. First, it accelerates drastically the convergence of the values of the $\mathcal{S}$-matrix elements, and second, they are needed in order to obtain the correct result.

\section{SOFT-CORE ${ }^{4} \mathrm{He}-{ }^{4} \mathrm{He}$ POTENTIAL AND THE ${ }^{4} \mathrm{He}^{4}{ }^{4} \mathrm{He}_{2}$ REACTION}

Now that the integral relations have been proved to be efficient to describe $1+2$ reactions above the breakup threshold, in this section we shall use them to study the atomic ${ }^{4} \mathrm{He}_{-}{ }^{4} \mathrm{He}_{2}$ process. In particular, we shall focus on three-body states with spin and parity $0^{+}$, and the possibility of using simple two-body soft-core potentials will be investigated.

The ${ }^{4} \mathrm{He}-{ }^{4} \mathrm{He}$ molecule is known to be one of the biggest diatomic molecules. Its binding energy has been estimated to be around $1 \mathrm{mK}$, with a scattering length $a$ around 190 a.u. $[12,13]$. Different accurate investigations of the ${ }^{4} \mathrm{He}-{ }^{4} \mathrm{He}$ interaction are available in the literature [14-17]. All of them present the common feature of a sharp repulsion below an interparticle distance of approximately 5 a.u. The presence of the repulsive core is the source of a series of important technical difficulties. For instance, the wave function in the inner regions, which is very small due to the large potential repulsion, is decisive for the energy of the bound states or the asymptotic properties of the continuum states. Therefore, the wave function must be calculated with high accuracy in this region, which typically requires a very important increase of the basis size. Furthermore, when using the adiabatic expansion method, the angular eigenvalues $\lambda_{n}(\rho)$ [see Eq. (3)] also diverge for small $\rho$, and this divergence provokes very frequent crossings between them that sometimes are not easy to handle [18].

In Ref. [9] the possibility of using a soft-core potential was investigated in the context of ${ }^{4} \mathrm{He}-{ }^{4} \mathrm{He}_{2}$ collisions below the threshold for breakup of the dimer. In particular the Gaussian potential suggested in [18],

$$
V_{2 b}(r)=-1.227 e^{-r^{2} / 10.03^{2}}
$$

was used (the strength of the potential is in $\mathrm{K}$ and the range is in a.u.).

The dimer properties obtained with this potential are given in the second column in the top of Table III. In particular, the dimer binding energy $E_{2 b}$, the two-body scattering length $a$, the effective range $r_{0}$, and the interatomic distance $\langle r\rangle$ are given. The two parameters in the soft-core Gaussian potential (26) were fitted to reproduce the scattering length and the effective range of the hard-core potential LM2M2 [14]. As seen in the third column of Table III (top part), when this is done the binding energy $E_{2 b}$ and the interatomic distance $\langle r\rangle$ are also well reproduced.

However, even if both potentials have the same two-body properties, when moving to the three-body states important differences appear. The soft-core potential overbinds the two bound states in ${ }^{4} \mathrm{He}_{3}$ and the atom-dimer scattering length is clearly smaller (second and fourth columns in the bottom part of Table III). In fact, as shown in [9], the phase shifts obtained with these two potentials for the ${ }^{4} \mathrm{He}_{-}{ }^{4} \mathrm{He}_{2}$ elastic scattering (below the breakup threshold) clearly differ from each other. As an example, for an incident energy of $1 \mathrm{mK}$, the phase shifts obtained with the Gaussian and the LM2M2 potentials are $-56^{\circ}$ and $-63^{\circ}$, respectively. 


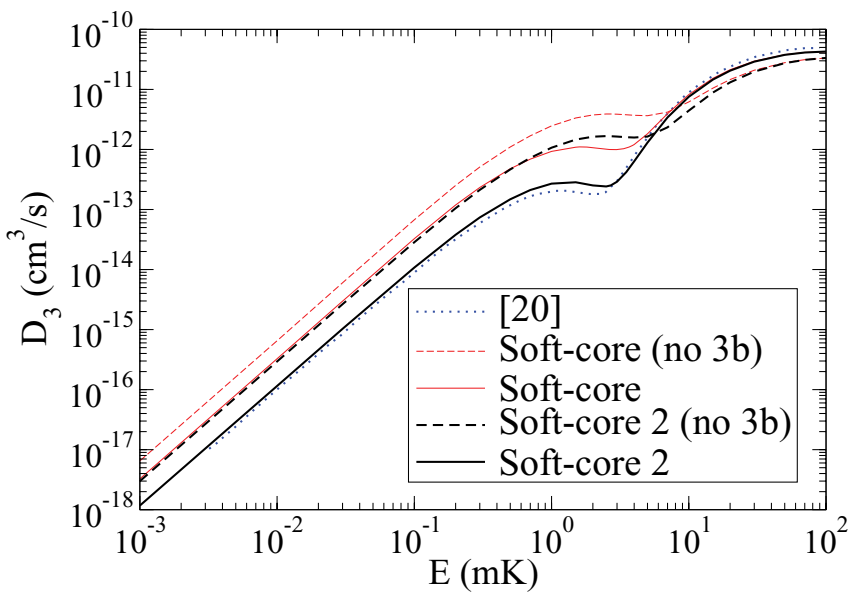

FIG. 3. (Color online) Dissociation rate [Eq. (28)] for the ${ }^{4} \mathrm{He}+$ ${ }^{4} \mathrm{He}_{2} \rightarrow{ }^{4} \mathrm{He}+{ }^{4} \mathrm{He}+{ }^{4} \mathrm{He}$ reaction as a function of the three-body energy $E$. The thin and thick curves have been obtained with soft-core potentials (26) and (30), respectively (see Table III). The dashed and solid curves are the results without and with the inclusion of an effective three-body force. The dotted curve is the result given in [20].

As also shown in [9], this anomalous behavior of the softcore potential at the three-body level can be corrected by using a short-range effective three-body force, depending only on the hyperradius, that is added to the effective potential (4). In particular we choose the simple three-body force

$$
W(\rho)=W_{0} e^{-\rho^{2} / \rho_{0}^{2}},
$$

where the strength $W_{0}$ is adjusted to reproduce the trimer ground-state binding energy obtained with the LM2M2 potential. When this is done the results are fairly independent of the range parameter $\rho_{0}$, at least within a reasonable value from $\rho_{0}=4$ a.u. to $\rho_{0}=10$ a.u. The results given in the third column (bottom part) of Table III have been obtained after inclusion of the three-body force. The precise values of $W_{0}$ and $\rho_{0}$ used are given in the first row of the bottom part of Table III. As we can see, once the binding energy of the ground state has been corrected, the binding energy of the excited state and the atom-dimer scattering length automatically agree with the corresponding values obtained with the LM2M2 potential. Furthermore, as seen in Fig. 3 of [9], the low-energy phase shifts are also corrected when the effective three-body force is included.

The importance of including the three-body force is also seen when investigating the ${ }^{4} \mathrm{He}^{4} \mathrm{He}_{2}$ reaction for incident energies $\left(E_{i}=E-E_{2 b}\right)$ above the threshold for breakup of the dimer, i.e., $E_{i}>\left|E_{2 b}\right|$ (or $E>0$ ). In Table IV we give the inelasticity $\left(\left|\mathcal{S}_{11}\right|\right)$ and the real part of the phase shift $\operatorname{Re}(\delta)$ for energies $E=5$ and $E=25 \mathrm{mK}$. As in Tables I and II, $K_{\max }$ is the grand-angular quantum number associated to the last adiabatic term included in the expansion (1). The values with no parentheses were when the three-body force was included in the calculation ( $W_{0}=18.314 \mathrm{~K}$ and $\rho_{0}=6$ a.u.), and the results within parentheses were obtained without the threebody force.

As we can see, the pattern of convergence is similar to the one observed in Tables I and II for the neutron-deuteron reaction. A $K_{\max }$ value of around 12 is enough to get a
TABLE IV. Inelasticity $\left|\mathcal{S}_{11}\right|$ and real part of the phase shift $\operatorname{Re}(\delta)$ for the ${ }^{4} \mathrm{He}^{-} \mathrm{He}_{2}$ collision at three-body energies (above threshold) $E=5 \mathrm{mK}$ and $E=25 \mathrm{mK}$. The value of $K_{\max }$ is the $K$ value associated with the last adiabatic potential included in the calculation. The numbers within parentheses have been obtained without inclusion of the three-body force.

\begin{tabular}{lccccc}
\hline \hline & \multicolumn{2}{c}{$\left|\mathcal{S}_{11}\right|$} & & \multicolumn{2}{c}{$\operatorname{Re}(\delta)$} \\
\cline { 2 - 3 } \cline { 5 - 6 }$K_{\text {max }}$ & $E=5 \mathrm{mK}$ & $E=25 \mathrm{mK}$ & & $E=5 \mathrm{mK}$ & $E=25 \mathrm{mK}$ \\
\hline 4 & $\mathbf{0 . 9 9 8 8}$ & $\mathbf{0 . 9 3 5 1}$ & & $\mathbf{6 9 . 3 0}$ & $\mathbf{3 5 . 0 9}$ \\
& $(0.9946)$ & $(0.9645)$ & & $(75.52)$ & $(40.59)$ \\
8 & $\mathbf{0 . 9 9 8 8}$ & $\mathbf{0 . 9 1 1 1}$ & & $\mathbf{6 9 . 2 3}$ & $\mathbf{3 4 . 8 0}$ \\
& $(0.9946)$ & $(0.9403)$ & & $(75.45)$ & $(40.28)$ \\
12 & $\mathbf{0 . 9 9 8 9}$ & $\mathbf{0 . 9 1 0 4}$ & & $\mathbf{6 9 . 2 0}$ & $\mathbf{3 4 . 6 3}$ \\
& $(0.9947)$ & $(0.9394)$ & & $(75.41)$ & $(40.09)$ \\
16 & $\mathbf{0 . 9 9 8 9}$ & $\mathbf{0 . 9 1 1 0}$ & & $\mathbf{6 9 . 1 7}$ & $\mathbf{3 4 . 6 0}$ \\
& $(0.9947)$ & $(0.9402)$ & & $(75.39)$ & $(40.05)$ \\
20 & $\mathbf{0 . 9 9 8 9}$ & $\mathbf{0 . 9 1 1 0}$ & & $\mathbf{6 9 . 1 6}$ & $\mathbf{3 4 . 5 9}$ \\
& $(0.9947)$ & $(0.9402)$ & & $(75.38)$ & $(40.04)$ \\
24 & $\mathbf{0 . 9 9 8 9}$ & $\mathbf{0 . 9 1 0 9}$ & & $\mathbf{6 9 . 1 5}$ & $\mathbf{3 4 . 5 8}$ \\
& $(0.9947)$ & $(0.9402)$ & & $(75.38)$ & $(40.04)$ \\
28 & $\mathbf{0 . 9 9 8 9}$ & $\mathbf{0 . 9 1 0 9}$ & $\mathbf{6 9 . 1 5}$ & $\mathbf{3 4 . 5 8}$ \\
& $(0.9947)$ & $(0.9402)$ & $(75.37)$ & $(40.04)$ \\
40 & $\mathbf{0 . 9 9 8 9}$ & $\mathbf{0 . 9 1 0 9}$ & $\mathbf{6 9 . 1 5}$ & $\mathbf{3 4 . 5 8}$ \\
& $(0.9947)$ & $(0.9402)$ & $(75.37)$ & $(40.04)$ \\
\hline \hline
\end{tabular}

rather well converged inelasticity, while $\operatorname{Re}(\delta)$ requires a few more adiabatic terms in order to reach convergence. Similar to what is found in [9] for energies below the breakup threshold, the inclusion of the three-body force gives rise to relevant changes in the computed values. These changes are particularly noticeable for $\operatorname{Re}(\delta)$, which for the two energies under consideration increase up to $6^{\circ}$ when the three-body force is not included in the calculation. Also, we observe that for $E=5 \mathrm{mK}$ the breakup probability $\left(1-\left|\mathcal{S}_{11}\right|^{2}\right)$ is still rather small, clearly smaller than $1 \%$ (with three-body force), while for $E=25 \mathrm{mK}$ this probability increases to $17 \%$ (12\% without three-body force).

With the $\mathcal{S}$ matrix in hand, we can now compute the dissociation rate for the ${ }^{4} \mathrm{He}_{-}{ }^{4} \mathrm{He}_{2}$ collision. The analytic form of this rate is given in Ref. [20], and for $0^{+}$states it becomes

$$
D_{3}=\frac{\hbar \pi}{\mu_{1,23} k_{1,23}}\left(1-\left|\mathcal{S}_{11}\right|^{2}\right)
$$

where

$$
\mu_{1,23}=\frac{2 M_{\mathrm{He}}}{3}, \quad k_{1,23}^{2}=\frac{2 \mu_{1,23} E_{i}}{\hbar^{2}},
$$

$M_{\mathrm{He}}$ is the mass of the ${ }^{4} \mathrm{He}$ atom, and $E_{i}=E-E_{2 b}$ is the incident energy in the center of mass frame ( $E_{2 b}$ is the binding energy of the ${ }^{4} \mathrm{He}_{2}$ dimer). In Fig. 3 we show the dissociation rate as a function of the three-body energy $E$. The thin dashed and thin solid lines are the results obtained with the Gaussian soft-core potential (26) without and with the additional threebody force (27), respectively. The low-energy behavior of the dissociation rate follows the $E^{K_{\mathrm{m}}+2}$ rule derived in [21], where $K_{\mathrm{m}}$ is the smallest grand-angular quantum number associated to the continuum adiabatic channels $\left(K_{\mathrm{m}}=0\right.$ in our case of three indistinguishable bosons coupled to $J^{\pi}=0^{+}$). 
From Fig. 3 we can see that the effect of the three-body force is quite important. In fact, for small energies the three-body force reduces the rate by a factor of 2. At higher energies, beyond $10 \mathrm{mK}$, the effect is the opposite, and the three-body force increases the rate by a factor close to 1.5 .

In Ref. [20] the same dissociation rate has been computed. The corresponding curve is shown in Fig. 3 by the dotted curve. As we can see, there is an important difference compared to our calculation. Except at high energies $(E \gtrsim 10 \mathrm{mK})$, where our result (with three-body force) and the one in [20] basically coincide, our rate is about a factor of 3 bigger.

However, we have to note that the hard-core two-body potential used in [20] gives rise to somewhat different dimer properties compared to the soft-core potential (26) and the LM2M2 potential [14]. In [20] they have used the potential based on the symmetry-adapted perturbation theory (SAPT) derived in [17]. The two-body properties obtained with this potential are given in the last column (top part) of Table III. The two-body dimer is $20 \%$ more bound than with the LM2M2 potential, and therefore the scattering length and the interatomic distance are smaller than in the LM2M2 case.

To investigate the sensitivity of the dissociation rate to the details of the two-body interaction we have constructed a second Gaussian soft-core potential reproducing the two-body properties of the SAPT potential. This potential takes the form

$$
V_{2 b}(r)=-1.234 e^{-r^{2} / 10.03^{2}},
$$

where the strength is given in $\mathrm{K}$ and the range is given in a.u. The corresponding two-body properties are given in the fourth column (top part) of Table III as soft core 2. Again, when moving to the three-body system, this new soft-core potential presents the same deficiencies as the previous one, i.e., overbinding of the three-body bound states and a too small atom-dimer scattering length. As before, this problem is solved by inclusion of the three-body force, whose parameters are again fitted to reproduce the ground-state binding energy of the helium trimer provided by the SAPT potential. The three-body properties with and without three-body force, as well as the parameters used for the three-body force, are given in the last three columns (bottom part) of Table III.

Making use of this new soft-core potential and the corresponding three-body force, we can compute again the dissociation rate (28). The results are shown in Fig. 3 by the thick dashed curve (no three-body force) and the thick solid curve (with three-body force). The new rates are now smaller than the ones obtained with the previous soft-core potential. Furthermore, when the three-body force is included and therefore not only the two-body properties but also the three-body ones agree with the ones obtained with the SAPT potential, our dissociation rate and the one given in [20] agree well for the whole range of energies. Only a small difference can be seen at about $1 \mathrm{mK}$. We can therefore see that the dissociation rate is very sensitive to the details of the two-body interaction. An additional $20 \%$ binding of the dimer and the corresponding decrease in the scattering length lead to a factor of 3 decrease in the rate.

The same conclusions are reached when investigating the recombination rate corresponding to the inverse process ${ }^{4} \mathrm{He}+$ ${ }^{4} \mathrm{He}+{ }^{4} \mathrm{He} \rightarrow{ }^{4} \mathrm{He}_{2}+{ }^{4} \mathrm{He}$. This rate is given by the same

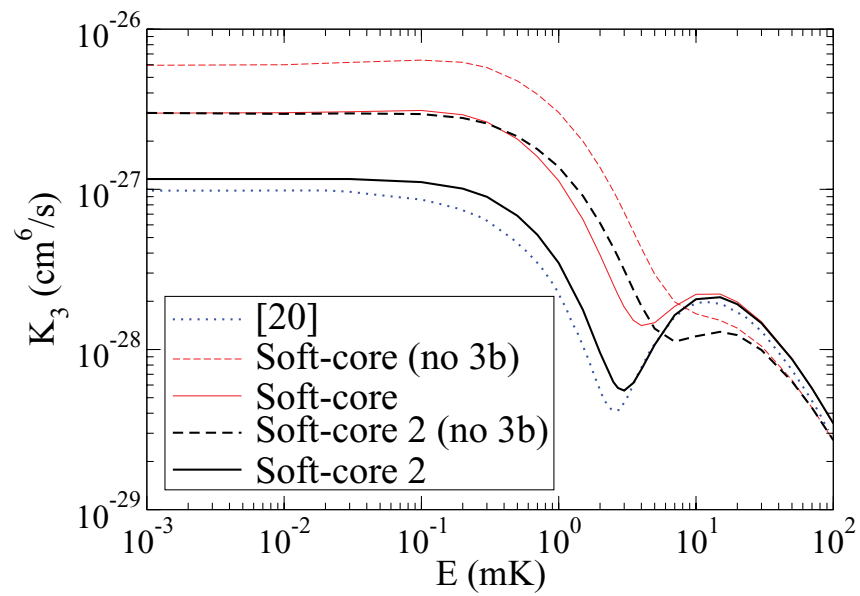

FIG. 4. (Color online) The same as Fig. 3 for the recombination rate [Eq. (31)] for the process ${ }^{4} \mathrm{He}+{ }^{4} \mathrm{He}+{ }^{4} \mathrm{He} \rightarrow{ }^{4} \mathrm{He}_{2}+{ }^{4} \mathrm{He}$.

$\mathcal{S}$-matrix elements as in the dissociation case, and its analytical form for three identical bosons with angular momentum and parity $0^{+}$is given by [20]

$$
K_{3}=3 ! \frac{32 \hbar \pi^{2}}{\mu k^{4}}\left(1-\left|\mathcal{S}_{11}\right|^{2}\right)
$$

where

$$
\mu^{2}=\frac{M_{\mathrm{He}}^{2}}{3}, \quad k^{2}=\frac{2 \mu E}{\hbar^{2}}
$$

$M_{\mathrm{He}}$ is the mass of the ${ }^{4} \mathrm{He}$ atom, and $E$ is the total three-body energy.

The computed recombination rate is shown in Fig. 4. The meaning of the curves is as in Fig. 3. In Ref. [21] the recombination rate $K_{3}$ was proved to behave at low energies as $E^{K \mathrm{~m}}\left(K_{\mathrm{m}}\right.$ is the smallest grand-angular quantum number associated with the continuum adiabatic channels), which means that in our case, where $K_{\mathrm{m}}=0, K_{3}$ should be constant at very low energies, as observed in Fig. 4. Again, inclusion of the three-body force reduces the rate by a factor of 2 to 3 at small energies (compare the dashed curves and the corresponding solid curves in Fig. 4). Also, when soft-core potential (30) together with the three-body force is used, the computed recombination rate agrees well with the rate given in [20] (dotted curve in Fig. 4). This fact reveals again the importance of the fine details of the two-body interaction.

Finally, it is important to emphasize that in this work the $\mathcal{S}$ matrix has been obtained by using integral relations (19) and (20). As explained, they have the great advantage of needing only the internal part of the wave functions. In fact, the integrals involved in the calculations shown in this section can be safely performed with a maximum value for the hyperradius $\rho$ of 4000 a.u. Even a smaller value could be enough. However, in [20], where the adiabatic expansion was also used, the radial wave functions in Eq. (1) had to be expanded up to $5 \times$ $10^{5}$ a.u., which is definitely very delicate from the numerical point of view. 


\section{SUMMARY AND CONCLUSIONS}

In this paper we have extended the use of the integral relations derived in [1,2] to describe $1+2$ reactions above the threshold for breakup of the dimer. As in [1,2], the integral relations are used in combination with the adiabatic expansion method in order to construct the trial wave function. The main consequence of moving up to energies above the breakup threshold is that the $\mathcal{S}$ matrix describing the process now has an infinite dimension. This was not the case for energies below the threshold, where the elastic, inelastic, or transfer channels were described by a finite (and small) number of adiabatic terms.

The applicability of the method has been tested with the neutron-deuteron reaction, for which benchmark calculations are available. The agreement with these calculations is good, and the pattern of convergence is similar to the one found in [1,2] for energies below the breakup threshold. The integral relations accelerate the convergence significantly, and typically, about 10 adiabatic terms are enough to get a rather well converged result.

The method was then used to investigate reactions involving three helium atoms. In particular we have focused on the possibility of using soft-core atom-atom potentials in order to describe the full process. These potentials permit us to avoid all the technical problems arising from the use of more sophisticated potentials where a hard-core repulsion is always present. However, as already shown in [9], even if the soft-core potentials reproduce the dimer properties properly, an effective three-body force is needed in order to reproduce as well the properties of the trimer bound states and the atom-dimer scattering length obtained with the hard-core potentials.

We have found that the three-body force also modifies significantly the inelasticity and the phase shift for energies above the breakup threshold. In fact, when computing the reaction rates for dissociation of the dimer $\left({ }^{4} \mathrm{He}+{ }^{4} \mathrm{He}_{2} \rightarrow\right.$ $\left.{ }^{4} \mathrm{He}+{ }^{4} \mathrm{He}+{ }^{4} \mathrm{He}\right)$ and for the recombination process $\left({ }^{4} \mathrm{He}+\right.$ ${ }^{4} \mathrm{He}+{ }^{4} \mathrm{He} \rightarrow{ }^{4} \mathrm{He}_{2}+{ }^{4} \mathrm{He}$ ), the three-body force decreases the rates even by a factor of 3 at low energies.

These two rates are also very sensitive to the details of the two-body interaction. We have found that a two-body potential, such as the SAPT potential, providing a dimer state about $20 \%$ more bound than the one obtained with the LM2M2 potential, also reduces the rates by a factor of around 3 at small energies. The soft-core potential reproducing the two-body properties of the SAPT potential, together with the corresponding threebody force designed to reproduce the trimer properties as well, gives then rise to reaction rates in very good agreement with the ones of the SAPT potential.

In summary, the integral relations are also useful in order to describe reactions above the breakup threshold. They accelerate significantly the convergence of the $\mathcal{S}$-matrix terms. For reactions involving hard-core two-body potentials, the use of soft-core potentials with the same two-body properties are a very good alternative, provided that they are used together with an effective three-body force designed to fit as well the bound state three-body energies. When this is done, the reaction rates obtained with the hard-core and the soft-core potentials agree pretty well. These rates are very sensitive to the details of the two-body interaction. Small variations of the dimer properties can produce sizable changes in the reaction rates at low energies.

\section{ACKNOWLEDGMENT}

This work was partly supported by funds provided by DGI of MINECO (Spain) under Contract No. FIS2011-23565.
[1] P. Barletta, C. Romero-Redondo, A. Kievsky, M. Viviani, and E. Garrido, Phys. Rev. Lett. 103, 090402 (2009).

[2] C. Romero-Redondo, E. Garrido, P. Barletta, A. Kievsky, and M. Viviani, Phys. Rev. A 83, 022705 (2011).

[3] F. E. Harris, Phys. Rev. Lett. 19, 173 (1967).

[4] A. R. Holt and B. Santoso, J. Phys. B 5, 497 (1972).

[5] A. Kievsky, M. Viviani, P. Barletta, C. Romero-Redondo, and E. Garrido, Phys. Rev. C 81, 034002 (2010).

[6] P. Barletta and A. Kievsky, Few Body Syst. 45, 25 (2009).

[7] J. L. Friar et al., Phys. Rev. C 42, 1838 (1990).

[8] J. L. Friar, G. L. Payne, W. Glöckle, D. Hüber, and H. Witala, Phys. Rev. C 51, 2356 (1995).

[9] A. Kievsky, E. Garrido, C. Romero-Redondo, and P. Barletta, Few Body Syst. 51, 259 (2011).

[10] F. M. Pen'kov, J. Exp. Theor. Phys. 97, 485 (2003).

[11] E. Nielsen, D. V. Fedorov, A. S. Jensen, and E. Garrido, Phys. Rep. 347, 373 (2001).
[12] F. Luo, C. F. Giese, and W. R. Gentry, J. Chem. Phys. 104, 1151 (1996).

[13] R. E. Grisenti, W. Schöllkopf, J. P. Toennies, G. C. Hegerfeldt, T. Köhler, and M. Stoll, Phys. Rev. Lett. 85, 2284 (2000).

[14] R. A. Aziz and M. J. Slaman, J. Chem. Phys. 94, 8047 (1991).

[15] K. T. Tang, J. P. Toennies, and C. L. Yiu, Phys. Rev. Lett. 74, 1546 (1995).

[16] T. Korona, H. L. Williams, R. Bukowski, B. Jeziorski, and K. Szalewicz, J. Chem. Phys. 106, 5109 (1997).

[17] M. Jeziorska, W. Cencek, K. Patkowsky, B. Jeziorski, and K. Szalewicz, J. Chem. Phys. 127, 124303 (2007).

[18] E. Nielsen, D. V. Fedorov, and A. S. Jensen, J. Phys. B 31, 4085 (1998).

[19] E. A. Kolganova, A. K. Motovilov, and W. Sandhas, Phys. Part. Nucl. 40, 206 (2009).

[20] H. Suno and B. D. Esry, Phys. Rev. A 78, 062701 (2008).

[21] B. D. Esry, C. H. Greene, and H. Suno, Phys. Rev. A 65, 010705 (2001). 